\title{
El cáncer de seno: conocimientos, actitudes y prácticas para la detección temprana en Amalfi, Antioquia
}

\author{
Breast cancer: Knowledge, attitudes and practices for early detection in Amalfi, \\ Antioquia
}

\author{
Câncer mamário: conhecimentos, atitudes e práticas para detecção prévia em \\ Amalfi, Antioquia
}

\begin{abstract}
Luis Fernando Rendón-Arango'; Simón Llano-Rendón²; Isabel C. Garcés-Palacio³.
1 Administrador en Salud, con énfasis en Gestión de servicios de salud. Seguros de Riesgos Laborales Suramericana S. A., Medellín, Colombia. Ifrendon1792@gmail.com. ORCID: https://orcid.org/0000-0003-0289-8822.

2 Administrador en Salud, con énfasis en Gestión de servicios de salud. Cooperativa de Hospitales de Antioquia. Medellín, Colombia. simon.llano.rendon@gmail.com. oRCID: https://orcid.org/0000-0001-6239-7590.

3 DrPH, MPH. Universidad de Antioquia. Medellín, Colombia. icristina.garces@udea.edu.co. oRCID: https://orcid.org/0000-0003-0531-276X.
\end{abstract}

Recibido: 22/02/2019. Aprobado: 15/05/2019. Publicado: 01/10/2019

Rendón-Arango LF, Llano-Rendón S, Garcés-Palacio IC. El cáncer de seno: conocimientos, actitudes y prácticas para la detección temprana en Amalfi, Antioquia. Rev. Fac. Nac. Salud Pública. 2019;37(3):5-14. Dor: 10.17533/udea.rfnsp.v37n3a02

\section{Resumen}

Objetivo: caracterizar los conocimientos sobre el cáncer de seno, y los saberes, las actitudes y las prácticas sobre su detección temprana en mujeres entre 30 y 69 años de edad del área urbana del municipio de Amalfi, Antioquia. Metodología: estudio de corte utilizando una encuesta con 335 mujeres, seleccionadas mediante muestreo aleatorio simple a partir de las direcciones de viviendas proporcionadas por la Secretaría de Planeación Municipal. Resultados: El puntaje combinado de conocimientos de cáncer de seno, factores de riesgo y síntomas fue 14 (desviación estándar: 3,6) de 22 puntos posibles, evidenciando un conocimiento intermedio en este tema. En mujeres entre 30 y 49 años, el 82, 14 y 68 \% sabían qué era el autoexamen, el examen clínico y la mamografía respectivametne, comparado con el 62,10 y $63 \%$ en mujeres entre 50 y 69 años. El $95 \%$ de las participantes reportó que los métodos de detección temprana son importantes para la prevención o el tratamiento oportuno del cáncer. El 71,9 \% de las participantes entre 30 y 49 años de edad, y el $67,1 \%$ entre 50 y 69 años se realizó el autoexamen de seno. El examen clínico de seno se lo hizo el 41,5\% de las mujeres, sin diferencias significativas entre los grupos de edad. Solamente el 39,2 \% de las mujeres entre los 50 y 69 años de edad se habían realizado la mamografía. Conclusiones: Los conocimientos sobre el cáncer de seno, y las actitudes y las prácticas de su detección temprana presentan deficiencias en las edades comparadas; sin embargo, existe una disposición favorable por parte de las mujeres hacia la detección temprana, que puede favorecer el fortalecimiento de conocimientos sobre este tipo de cáncer y motivar la práctica de su detección.

---------Palabras clave: cáncer de seno, diagnóstico de cáncer de seno, detección precoz del cáncer, Colombia. 


\begin{abstract}
Objective: To characterize informed and traditional knowledge on breast cancer, as well as attitudes, and practices for its early detection among 30 to 69 years old women in the urban area of the municipality of Amalfi, Antioquia. Methodology: Crosssectional study using a survey involving 335 women, selected via simple random sampling using housing addresses provided by the Secretariat for City Planning. Results: The combined score for breast cancer knowledge, risk factors, and symptoms was 14 (standard deviation: 3.6) out of 22 possible points, which shows an intermediate understanding of the topic. In 30 to 49 years old women, $82 \%$ of them knew what breast cancer self-examination was, $14 \%$ knew what clinical breast cancer exams were, and $68 \%$ knew what a mammography was. On the contrary, in 50 to 69 years old women, percentages for the same criteria were $62 \%, 10 \%$, and $63 \%$, respectively. $95 \%$ of the respondents
\end{abstract}

reported that early detection methods are important for cancer prevention or its timely treatment. $71.9 \%$ of the 39 to 49 years old respondents, as well as $67.1 \%$ of 50 to 69 years old women had already performed breast self-examination. $41.5 \%$ of women had taken a clinical breast exam, without significant differences between age groups. Only $39.2 \%$ of 50 to 69 years old women, had had a mammography. Conclusions: Knowledge on breast cancer, as well as attitudes, and practices for its early detection show deficiencies among the compared age groups; however, women present a favorable disposition towards breast cancer early detection, which may favor a strengthening of knowledge about this type of cancer and engage women in involving themselves in procedures for its detection.

-Keywords: breast cancer, breast cancer diagnosis, early cancer detection, Colombia.

\section{Resumo}

Objetivo: Caracterizar os saberes relacionados ao câncer mamário e os conhecimentos, atitudes e práticas referidas à detecção antecipada nas mulheres entre 30 e 69 anos na área intra urbana do município de Amalfi, Antioquia. Metodologia: estudo de corte utilizando uma enquete com 335 mulheres, escolhidas por amostragem arbitrária simples baseada nos endereços de vivendas fornecidos pela Secretaria Municipal de Planejamento. Resultados: $\mathrm{O}$ resultado misturado desses saberes, fatores de risco e sintomas do câncer de mama foi de 14 (desvio padrão: 3,6) dos 22 pontos possíveis, evidenciando um conhecimento intermediário nesse tópico. Nas mulheres entre os 30 e 49 anos, o 82, 14 e 68\% conheciam muito bem o que era o auto-exame, o exame clínico e uma mamografia, respectivamente, comparado com o 62, 10 e $63 \%$ em mulheres entre 50 e 69 anos. O 95\% dos participantes reportaram que os métodos de detecção antecipados são trascendentes para a prevenção ou para o tratamento oportuno do câncer. O 71,9\% das participantes entre 30 e 49 anos e o $67,1 \%$ entre 50 e 69 anos, fizeram o auto-exame das mamas. $\mathrm{O}$ exame clínico das mamas foi feito por o $41,5 \%$ das mulheres, sem diferenças significativas entre as faixas etárias. Somente o 39,2\% das mulheres entre 50 e 69 anos fizeram mamografia. Conclusões: Os conhecimentos sobre o câncer de mama e as atitudes e práticas de sua detecção precoce apresentam falhas nas idades comparadas; no entanto, existe uma disposição favorável das mulheres para a detecção precoce, o que pode favorecer o robustecimento do saber relacionado com esse tipo de câncer e motivar a prática de sua detecção.

-Palavras-chave: câncer de mama, diagnóstico de câncer de mama, detecção precoce de câncer, Colômbia.

\section{Introducción}

El cáncer de seno es una enfermedad de gran impacto a nivel mundial, ya que, en términos de incidencia, en el año 2018 ocupó el segundo lugar entre todos los cánceres, luego del de pulmón. En términos de mortalidad, fue la quinta causa de muerte por cáncer en general, y la causa más común de muerte en mujeres, incluyendo Colombia, con una incidencia ajustada de 44,1/100 000 mujeres y mortalidad de 11,9/100 000 [1]. Para el departamento de Antioquia, los datos disponibles revelan una tasa de mortalidad anual entre 2007 y 2013 de 10,3/100 000 [2].

Los conocimientos actuales sobre las causas del cáncer de seno son limitados, por lo que la detección temprana sigue siendo la piedra angular de la lucha contra esta enfermedad. La principal forma de detectar el cáncer de seno en estadios tempranos es a través de la mamografía. Sin embargo, según la "Encuesta Nacional de Demografía y Salud" (ENDS) de 2015, en Antioquia, excluyendo Medellín (su ciudad capital), solo el $33 \%$ de las mujeres se han practicado la mamografía, en gran parte por desconocimiento y falta de infraestructura fuera de ciudades capitales [3]. Esto obliga a acudir a modos de detección temprana menos efectivos, como el autoexamen y el examen clínico del seno, pues para muchas mujeres es la única alternativa de detección.

Los datos presentados por la ENDS reflejan el alto desconocimiento de los exámenes de detección. Esta falta de conocimiento, sumado a actitudes o creencias relacionadas con las pruebas de tamizaje, pueden generar una baja utilización de estas, como lo señalan varios artículos recientes realizados en diferentes partes del mundo [4-6]. Es por esto por lo que evidenciar los 
conocimientos acerca del cáncer de seno, y los saberes, las actitudes y las prácticas que tienen las mujeres sobre su detección temprana, puede ayudar a mejorar las acciones de prevención de esta enfermedad. Sin embargo, la información al respecto en mujeres colombianas de áreas rurales y pequeñas zonas urbanas es limitada. Amalfi, un municipio ubicado al nordeste del departamento de Antioquia, a unos $140 \mathrm{~km}$ de Medellín, no es ajeno a esta falta de información necesaria para la toma de decisiones, que puede ser de vital importancia para aseguradores en salud y autoridades sanitarias locales.

Dadas las altas tasas de incidencia y mortalidad del cáncer de seno en el país y por el interés de las autoridades sanitaras locales de Amalfi por tener mayor información para la toma de decisiones, nuestro objetivo fue describir los conocimientos sobre el cáncer de seno, y los saberes, las actitudes y las prácticas sobre su detección temprana en mujeres del área urbana del municipio de Amalfi, Antioquia.

La información encontrada también puede ser de utilidad para otras localidades con situaciones geográficas y culturales similares.

\section{Metodología}

Se llevó a cabo un estudio de corte, con mujeres entre los 30 y 69 años de edad residentes en el área urbana del municipio de Amalfi. Se escogió dicho grupo de edad debido a que el "Manual para la detección temprana de cáncer de mama", del Ministerio de la Protección Social de Colombia, recomienda acciones de detección en mujeres premenopáusicas y hasta los 69 años de edad [7]. Se calculó un tamaño de muestra de 335 mujeres, esperando encontrar una frecuencia del $50 \%$ en prácticas de detección temprana, con un nivel de confianza del $95 \%$ y un margen de error del $5 \%$. La selección se realizó mediante un muestreo aleatorio simple de todas las viviendas del área urbana del municipio, escogiendo al azar solamente una mujer en cada vivienda. Las direcciones de las viviendas fueron proporcionadas por la Secretaría de Planeación Municipal.

Las encuestas se realizaron durante el mes de febrero del año 2017, con una duración aproximada de 20 minutos por encuesta. Esta estaba constituida por 61 preguntas elaboradas por los investigadores con base en estudios previos reportados en la literatura [3,8]. Las preguntas estaban diseñadas con el objetivo de identificar los conocimientos generales que tenían las mujeres acerca de esta enfermedad, los síntomas y los factores de riesgo, al igual que el conocimiento que tuvieran acerca del autoexamen, el examen clínico y la mamografía, la disposición para su realización, periodicidad y la puesta en práctica de estos de acuerdo con la recomendación para su edad. El autoexamen se recomienda en mujeres pre- y posmenopausicas; el examen clínico del seno, para mujeres mayores de 40 años, y la mamografía, en mujeres entre 50 y 69 años de edad [7].

La encuesta fue sometida a un proceso de validación de apariencia con tres expertas en salud sexual y reproductiva. Luego de hacerle ajustes, la encuesta se aplicó a cuatro mujeres con el rango de edad estipulado en el proyecto. Con esta validación se realizaron ajustes, eliminando o modificando algunas preguntas para mayor claridad. Posteriormente, durante el entrenamiento a los encuestadores, el equipo de trabajo identificó algunas preguntas que también debieron ser modificadas, en aras de un mayor entendimiento y agilidad en el proceso de encuesta. Finalmente, se efectuó una prueba piloto en campo con veinticinco encuestadas, en donde se aprobaron los procedimientos de recolección y la claridad del cuestionario.

El conocimiento acerca del cáncer de seno y sus factores de riesgo, y sobre los síntomas se evaluó mediante 12 y 10 preguntas, respectivamente. Se creó un puntaje sumatorio con cada conjunto de preguntas, asignando un punto a todas las respuestas correctas y cero a las incorrectas. Por lo tanto, para conocimientos acerca del cáncer de seno y sus factores de riesgo, las participantes podrían tener puntajes entre 0 y 12 , y para conocimientos acerca de síntomas, entre 0 y 10 . Posteriormente, se sumaron los dos puntajes, para obtener un puntaje total de conocimiento que podría oscilar entre 0 (ningún conocimiento) y 22 (respondió todas las preguntas correctamente); luego, los puntajes se dividieron en bajo conocimiento (entre 0 y 7 ), medio (entre 8 y 15) y alto (entre 16 y 22).

A las participantes se les preguntó si sabían qué era el autoexamen de seno, el examen clínico del seno y la mamografía; a aquellas que refirieron no saber, se les leyó una descripción de dicho examen. A todas las participantes se les preguntó en qué rango de edad se deberían realizar estos exámenes, su periodicidad y posibles beneficios; también si se lo habían realizado, cuándo fue la última vez y con qué periodicidad los efectúa.

Se llevaron a cabo análisis bivariados entre cada una de las variables de desenlace: la práctica del autoexamen, el examen clínico o la mamografía, todas medidas como variables dicotómicas (sí, no), tomando como "sí", si se lo habían realizado al menos una vez en la vida; y las variables independientes, utilizando la prueba de $\mathrm{Chi}^{2}$. Las variables independientes fueron: estado civil (soltera, casada, unión libre, separada/ divorciada/viuda), afiliación al sistema de salud (no tiene, contributivo, subsidiado), número de hijos, haber tenido al menos un familiar con cáncer de seno, el puntaje sumatorio de conocimientos acerca del cáncer, factores de riesgo y síntomas, y grado de escolaridad — como 
variable ordinal con cinco niveles: ninguna o primaria incompleta, primaria completa, bachillerato incompleto, bachillerato completo, estudios superiores-. También se realizaron tres modelos de regresión logística multivariados (uno para cada desenlace), utilizando el método condicional hacia atrás, ingresando todas las variables independientes en cada uno de los modelos. Para todos los análisis se usó el paquete estadístico SPSS ${ }^{\circledR}$ versión 23 , de IBM.

El Comité de Ética de la Facultad Nacional de Salud Pública, de la Universidad de Antioquia, aprobó el 22 de diciembre de 2016, según el Acta 156 del 6 de diciembre, la realización del proyecto y lo catalogó como de riesgo mínimo. La investigación se desarrolló teniendo en cuenta la confidencialidad de la información y para tal fin se firmó un acta de confidencialidad entre encuestadores e investigadores. También se contó con un consentimiento informado escrito, que cumplió con las características descritas en el artículo 15 de la Resolución 8430 de 1993 [9], el cual fue aplicado por el encuestador, quien hizo entrega de una copia a la participante.

\section{Resultados}

Se encuestaron 335 mujeres, 57,3\% de ellas entre los 30 y 49 años de edad, y el 42,7\% restantes de 50 a 69 años. Entre las características sociodemográficas más representativas, se evidenció que el 35,5 \% no estudio o no terminó la primaria, y solamente 12,2 \% tenía estudios superiores. La mayoría (64,2 \%) destinaba su tiempo a las labores del hogar y el 32,8 \% trabajaba; adicionalmente, el 66,6 \% manifestó estar afiliado al régimen subsidiado del sistema de salud, y el 31,9\%, al régimen contributivo. En la Tabla 1 se presenta una descripción más detallada de la información sociodemográfica.

\section{Conocimientos del cáncer de seno y sobre su detección temprana}

Con relación al cáncer de seno, solo cuatro mujeres manifestaron no haber escuchado de este y el $21 \%$ de las participantes reportó haber tenido algún familiar diagnosticado con la enfermedad. El 91,3\% de las

Tabla 1. Información sociodemográfica de mujeres del área urbana del municipio de Amalfi.

\begin{tabular}{|c|c|c|c|c|c|c|c|}
\hline & \multirow{4}{*}{ Variables } & \multirow{2}{*}{\multicolumn{2}{|c|}{ Total }} & \multicolumn{4}{|c|}{ Edad } \\
\hline & & & & \multicolumn{2}{|c|}{ 30-49 años } & \multicolumn{2}{|c|}{$50-69$ años } \\
\hline & & \multicolumn{2}{|c|}{$N=335$} & \multicolumn{2}{|c|}{$\mathrm{n}=192$} & \multicolumn{2}{|c|}{$n=143$} \\
\hline & & $n$ & $\%$ & $n$ & $\%$ & $n$ & $\%$ \\
\hline \multirow{5}{*}{ Educación } & Ninguna o primaria incompleta & 119 & 35,5 & 43 & 22,4 & 76 & 53,1 \\
\hline & Primaria completa & 55 & 16,5 & 31 & 16,2 & 24 & 16,8 \\
\hline & Bachillerato incompleto & 40 & 11,9 & 25 & 13,0 & 15 & 10,5 \\
\hline & Bachillerato completo & 80 & 23,9 & 62 & 32,3 & 18 & 12,6 \\
\hline & Técnico/Tecnológica/Universitaria & 41 & 12,2 & 31 & 16,1 & 10 & 7,0 \\
\hline \multirow{4}{*}{ Estado civil } & Soltera & 98 & 29,3 & 58 & 30,2 & 40 & 28,0 \\
\hline & Casada & 106 & 31,6 & 59 & 30,7 & 47 & 32,8 \\
\hline & Unión libre & 81 & 24,2 & 62 & 32,3 & 19 & 13,3 \\
\hline & Separada/Divorciada/Viuda & 50 & 14,9 & 13 & 6,8 & 37 & 25,9 \\
\hline \multirow{4}{*}{ Ocupación } & Ama de casa & 215 & 64,2 & 112 & 58,3 & 103 & 72,0 \\
\hline & Estudiante/jubilada & 10 & 3,0 & 5 & 2,6 & 5 & 3,5 \\
\hline & Trabajadora dependiente & 36 & 10,7 & 26 & 13,6 & 10 & 7,0 \\
\hline & Trabajadora independiente & 74 & 22,1 & 49 & 25,5 & 25 & 17,5 \\
\hline \multirow{3}{*}{ Afiliación en salud } & No tiene & 5 & 1,5 & 2 & 1,0 & 3 & 2,1 \\
\hline & EPS contributiva & 107 & 31,9 & 62 & 32,3 & 45 & 31,5 \\
\hline & EPS subsidiada & 223 & 66,6 & 128 & 66,7 & 95 & 66,4 \\
\hline Número de hijos & Media (desviación estándar) & 3,0 & 2,431 & 2,2 & 1,334 & 4,1 & 3,062 \\
\hline
\end{tabular}


mujeres afirma que el cáncer de seno es curable si se detecta en forma temprana.

Sin embargo, más del $75 \%$ de las participantes respondió de manera incorrecta las preguntas relacionadas con los factores de riesgo hormonales, como menarquia antes de los 12 años, menopausia después de los 52 años y no tener hijos, las cuales tuvieron 79,7, 80,1 y $77 \%$ de respuestas incorrectas respectivamente.

El puntaje promedio de conocimientos generales sobre cáncer de seno y sus factores de riesgo fue 6,6 (desviación estándar: 1,96), de 12 puntos posibles. No hubo diferencias en los puntajes entre los grupos de edad. La Figura 1 muestra las respuestas para los factores de riesgo indagados.
En general, el conocimiento acerca de síntomas del cáncer de seno fue adecuado, con un puntaje promedio de 7,6 (desviación estándar: 2,5), de 10 puntos posibles.

Dos síntomas en particular fueron menos conocidos: casi la mitad de las participantes no reconocieron los bultos alrededor del cuello, y la piel del seno hundida o pezón hundido como un síntoma.

La Figura 2 presenta los síntomas indagados. El puntaje combinado de conocimientos de cáncer de seno, factores de riesgo y síntomas fue 14,0 (desviación estándar: 3,6), de 22 puntos posibles, evidenciando un conocimiento intermedio en este tema.

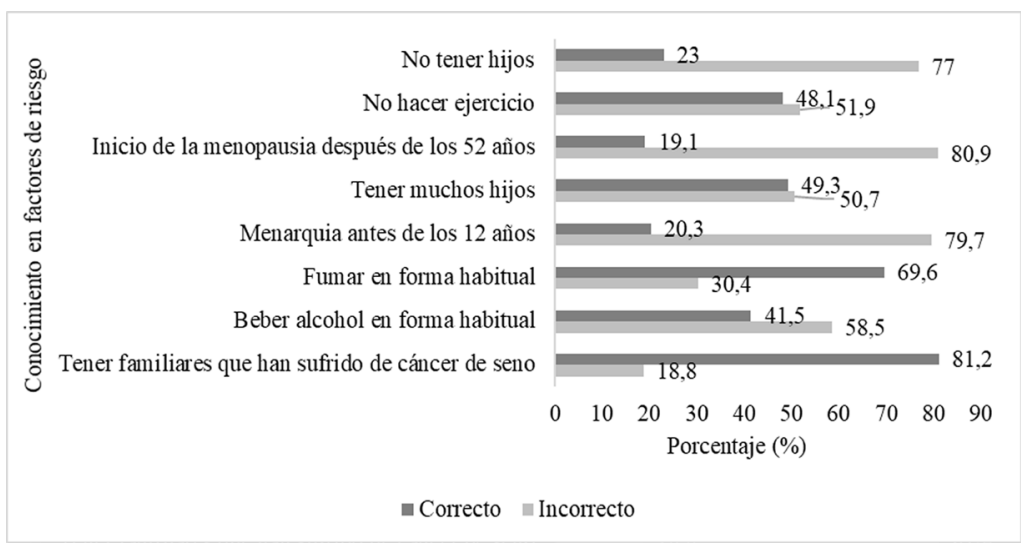

Figura 1. Conocimientos acerca de los factores de riesgo para cáncer de seno en mujeres entre 30 y 69 años de edad, del área urbana del municipio de Amalfi.

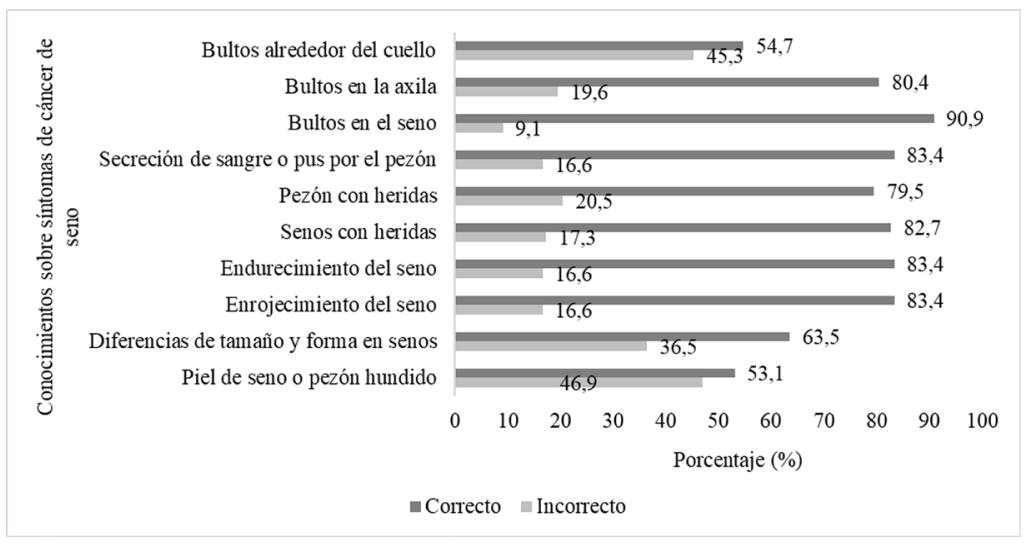

Figura 2. Conocimientos acerca de los síntomas del cáncer de seno en mujeres entre 30 y 69 años de edad, del área urbana del municipio de Amalfi. 
Al indagar si las participantes sabían qué eran el autoexamen de seno, el examen clínico de seno y la mamografía, se encontró que las de 30 a 49 años conocían más acerca de estas estrategias de prevención que aquellas entre los 50 y 69 años. En el grupo de mujeres más jóvenes, el 82,14 y $68 \%$ sabían qué eran el autoexamen, el examen clínico y la mamografía respectivamente, respecto al 62,10 y $63 \%$, respectivamente, de las mujeres del grupo de edad más avanzado. Se evidencia un marcado desconocimiento acerca del examen clínico del seno y solo el $66 \%$ del total de las participantes reportó saber qué es la mamografía, evidenciándose un conocimiento más bajo en el grupo de mujeres que debía realizarse dicho examen.
El 80,3\% de las encuestadas manifiesta saber cómo se efectúa el autoexamen; sin embargo, el conocimiento acerca de la frecuencia (que debe ser mensualmente) y el momento ideal del mes para llevarlo a cabo (5 a 7 días después del periodo menstrual) fue bajo. De manera similar, el conocimiento sobre el examen clínico de seno y la mamografía, en particular sobre las edades y la frecuencia de realización fue limitado. Por ejemplo, el 97,6 \% de las participantes desconocían la frecuencia con la que se debía hacer la mamografía y más del $90 \%$ desconocían las edades de realización de dicho examen, que en su caso les corresponde, al examen clínico, realizarlo cada 3 años a partir de los 20 años, y cada año, después de los 40 años; y para la mamografía, cada 2 años a las mujeres que estén por encima de los 50 años. (véase Tabla 2).

Tabla 2. Conocimientos acerca de los métodos de detección temprana en mujeres entre 30 y 69 años del área urbana del municipio de Amalfi.

\begin{tabular}{|c|c|c|c|c|c|c|c|}
\hline \multicolumn{2}{|l|}{ Conocimientos } & \multicolumn{2}{|c|}{ Total } & \multicolumn{2}{|c|}{ 30-49 años } & \multicolumn{2}{|c|}{ 50-69 años } \\
\hline 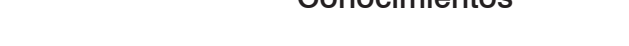 & & $\mathrm{n}$ & $\%$ & $\mathrm{n}$ & $\%$ & $\mathrm{n}$ & $\%$ \\
\hline \multirow[t]{2}{*}{ ¿Sabe cómo se realiza el autoexamen de seno? } & Sí & 269 & 80,3 & 163 & 84,9 & 106 & 74,1 \\
\hline & No & 66 & 19,7 & 29 & 15,1 & 37 & 25,9 \\
\hline \multirow{2}{*}{$\begin{array}{l}\text { ¿Cada cuánto se debe realizarse el } \\
\text { autoexamen? }\end{array}$} & Correcto & 131 & 39,1 & 91 & 47,4 & 40 & 28 \\
\hline & Incorrecto & 204 & 60,9 & 101 & 52,6 & 103 & 72 \\
\hline \multirow{2}{*}{$\begin{array}{l}\text { ¿En qué momento del mes se debe realizar el } \\
\text { autoexamen? }\end{array}$} & Correcto & 94 & 28,1 & 63 & 32,8 & 31 & 21,7 \\
\hline & Incorrecto & 241 & 71,9 & 129 & 67,2 & 112 & 78,3 \\
\hline \multirow{2}{*}{$\begin{array}{l}\text { ¿A partir de qué edad se debe realizar el } \\
\text { examen clínico de seno? }\end{array}$} & Correcto & 44 & 13,1 & 23 & 12 & 21 & 14,7 \\
\hline & Incorrecto & 291 & 86,9 & 169 & 88 & 122 & 85,3 \\
\hline \multirow{2}{*}{$\begin{array}{l}\text { ¿Hasta qué edad se debe realizar el examen } \\
\text { clínico de seno? }\end{array}$} & Correcto & 102 & 30,4 & 53 & 27,6 & 49 & 34,3 \\
\hline & Incorrecto & 233 & 69,6 & 139 & 72,4 & 94 & 65,7 \\
\hline \multirow{2}{*}{$\begin{array}{l}\text { ¿Con que frecuencia se debe realizar el examen } \\
\text { clínico de seno? }\end{array}$} & Correcto & 118 & 35,2 & 62 & 32,3 & 56 & 39,2 \\
\hline & Incorrecto & 217 & 64,8 & 130 & 67,7 & 87 & 60,8 \\
\hline \multirow{2}{*}{$\begin{array}{l}\text { ¿A partir de qué edad se debe realizar la } \\
\text { mamografía? }\end{array}$} & Correcto & 29 & 8,7 & 13 & 6,8 & 16 & 11,2 \\
\hline & Incorrecto & 306 & 91,3 & 179 & 93,2 & 127 & 88,8 \\
\hline \multirow{2}{*}{$\begin{array}{l}\text { ¿Hasta qué edad se debe realizar la } \\
\text { mamografía? }\end{array}$} & Correcto & 1 & 0,3 & 0 & 0 & 1 & 0,7 \\
\hline & Incorrecto & 334 & 99,7 & 192 & 100 & 142 & 99,3 \\
\hline \multirow{2}{*}{$\begin{array}{l}\text { ¿Con que frecuencia se debe realizar la } \\
\text { mamografía? }\end{array}$} & Correcto & 8 & 2,4 & 4 & 2,1 & 4 & 2,8 \\
\hline & Incorrecto & 327 & 97,6 & 188 & 97,9 & 139 & 97,2 \\
\hline
\end{tabular}

\section{Actitudes frente al cáncer de seno y su detección temprana}

Con relación a los beneficios de los métodos de detección temprana, la mayoría de participantes reportó acertadamente que estos son importantes para la prevención o el tratamiento oportuno del cáncer. Solo el 4,8, 7,2 y $9 \%$ de las participantes consideraron erróneamente que el autoexamen, el examen clínico y la mamografía, respectivamente, no tienen ningún beneficio.

\section{Prácticas de detección temprana del cáncer de seno}

El $71,9 \%$ de las participantes entre los 30 y 49 años de edad, y el $67,1 \%$ entre los 50 y 69 años se realizaron el autoexamen de seno. El examen clínico de seno se lo hizo el $41,5 \%$ de las mujeres, sin diferencias significativas entre los grupos de edad. Solo el 39,2 \% de las mujeres entre los 50 y 69 años se había efectuado la mamografía. Adicionalmente, el 17,2\% de las del grupo de edad más joven también se la habían realizado. 
Entre las razones más comunes para no hacerse el autoexamen están, con un 36,6\%, el olvido, pereza o descuido, seguido por la falta de síntomas, con el 35,6\%. Asimismo, no tener síntomas fue la principal razón para no llevar a cabo el examen clínico de seno, con un $60,7 \%$, seguido de la pereza, olvido o descuido, con un $19,8 \%$, y un $11,7 \%$ manifestó no habérselo realizado porque el médico no se los había recomendado.

Las participantes podían reportar varias razones para no hacerse la mamografía: el $85 \%$ de las mujeres entre 50 y 69 años, para las cuales está recomendado este tamizaje, mencionó entre 0 y 4 razones. Las que más respuestas positivas presentaron fueron no tener síntomas ( $92 \%$ ) y no haber recibido una recomendación médica para hacérsela $(70,1 \%)$, seguido por falta de dinero (34,5\%), porque la Entidad Prestadora de Salud (EPS) no la autoriza $(28,7 \%)$, temor a tener algo mal $(24,1 \%)$ y no estar en edad para hacérsela $(20,7 \%)$. Las razones de falta de tiempo, distancia del servicio de salud y dolor del procedimiento, cada una tuvo el 19,5\% de respuestas positivas, mientras la vergüenza fue mencionada como razón para no hacerse la mamografía por $14,9 \%$ de las participantes.

En el $69,6 \%$ de mujeres entre los 50 y 69 años de edad, la última mamografía fue ordenada por el médico general, el 23,2\% por un ginecólogo y 7,1\% por otro especialista. Las EPS pagaron la mamografía en su totalidad a $59,6 \%$ de las participantes, a $2,4 \%$ se les pagó parcialmente y $37,1 \%$ asumieron el costo; no hubo diferencias significativas entre grupos de edad. Además, el 96,6 \% de las mujeres de 50 a 69 años de edad han tenido que asumir en su totalidad los gastos de transporte, y el $89,3 \%$, los gastos de hospedaje. Nueve mujeres requirieron tratamiento después de la mamografía y todas recibieron dicho tratamiento.

Del total de participantes, $52,6 \%$ de las mujeres entre 30 y 49 años, y el $60,8 \%$ de las mujeres entre 50 y 69 años han pensado en hacerse una mamografía en el próximo año. De las 89 participantes que se han hecho la mamografía, el $72,7 \%$ de las mujeres entre 30 y 49 años, y el 76,8 \% de las que tienen entre 50 y 69 años manifestaron haber pensado en hacerse otra mamografía en algún momento de sus vidas.

En las mujeres entre 50 y 69 años de edad, el $69,5 \%$ afiliadas al régimen de salud subsidiado, el $44,4 \%$ del régimen contributivo, y el 33,3\% de las no afiliadas no se habían efectuado la mamografía y estas diferencias fueron estadísticamente significativas (valor $p=0,011$ ). En las mujeres entre 30 y 49 años no se encontraron diferencias estadísticamente significativas de acuerdo con el régimen de afiliación. En el caso del examen clínico del seno, aunque las mujeres del régimen contributivo reportaron en mayor proporción la práctica (48,6\% del régimen contributivo y $37,7 \%$ del régimen subsidiado), no hubo diferencias estadísticamente significativas para ninguno de los grupos etarios. En cuanto al autoexamen de seno, hubo mayor práctica en las mujeres del régimen contributivo $(82,2 \%)$ y en las que no tenían afiliación $(80,0 \%$ ), que en las afiliadas al régimen subsidiado $(63,7 \%)$ (valor $p=0,002)$; los datos son similares en los dos grupos etarios.

\section{Factores relacionados con los exámenes de detección temprana}

Se analizó la práctica del autoexamen, el examen clínico y la mamografía de acuerdo con categorías de nivel educativo y se encontró que las participantes con menos educación fueron las que menos se hicieron los exámenes de detección temprana, diferencias que son estadísticamente significativas en las mujeres entre 50 y 69 años. Para las mujeres entre 30 y 49 años se hallaron diferencias estadísticas solamente en el autoexamen. Específicamente para la mamografía, entre las mujeres de 50 a 69 años de edad, las que en mayor proporción mencionaron no realizarse la mamografía fueron las que no tenían ningún nivel educativo o tenían primaria incompleta (75\%), seguidas por aquellas que habían terminado la primaria (54\%), y continúa disminuyendo hasta el $30 \%$ en las mujeres con estudios superiores. Esta diferencia fue estadísticamente significativa (valor $p=0,004$ ).

Los modelos multivariados reducidos confirman la asociación enunciada (véase Tabla 3). Comparado con mujeres con menores niveles educativos, las mujeres con mayor educación de todas las edades eran más dadas a realizarse el autoexamen, y aquellas entre los 50 y 69 años eran más dadas a hacerse el examen clínico del seno y la mamografía. Adicionalmente, para las mujeres entre los 30 y 49 años de edad, el tener una familiar diagnosticada con cáncer de seno se asoció a una mayor práctica del examen clínico del seno y la mamografía.

\section{Discusión}

Este estudio permitió evidenciar que alrededor del 69,9 y el $41,5 \%$ de las mujeres de la zona urbana de Amalfi se habían realizado el autoexamen y el examen clínico del seno, respectivamente, y entre las mujeres de 50 a 69 años, solo el 39,2 \% se había hecho la mamografía. Se presentó un conocimiento intermedio con relación al cáncer de seno, sus factores de riesgo y síntomas. Sin embargo, el conocimiento acerca de las edades y la frecuencia de realización de los exámenes de detección temprana es bastante limitado. La práctica de la mamografía fue mayor en las mujeres sin afiliación al sistema de salud (67\%), seguidas por las del régimen contributivo $(56 \%)$ y tan solo el $31 \%$ del régimen subsidiado. Entre las razones más importantes para no hacerse los exámenes de detección temprana se 
Tabla 3. Modelos reducidos de regresión logística de factores asociados con la práctica del autoexamen, examen clínico del seno y mamografía, estratificados por edad en mujeres del área urbana del municipio de Amalfi.*

\begin{tabular}{|c|c|c|c|c|c|c|}
\hline \multirow[t]{2}{*}{ Tipo de examen } & \multirow{2}{*}{$\begin{array}{l}\text { Edad } \\
\text { (años) }\end{array}$} & \multirow{2}{*}{\multicolumn{2}{|c|}{ Factores asociados }} & \multirow[t]{2}{*}{ OR } & \multicolumn{2}{|c|}{$\begin{array}{l}\text { Intervalo de confi- } \\
\text { anza } 95 \%\end{array}$} \\
\hline & & & & & Bajo & Alto \\
\hline \multirow{6}{*}{ Autoexamen } & \multirow{4}{*}{$30-49$} & \multirow{3}{*}{$\begin{array}{l}\text { Conocimientos de } \\
\text { cáncer de mama }\end{array}$} & Conocimiento bajo & 0,17 & 0,04 & 0,76 \\
\hline & & & Conocimiento intermedio & 0,49 & 0,24 & 1,01 \\
\hline & & & Conocimiento alto & 1,0 & & \\
\hline & & Educación & & 1,55 & 1,16 & 2,07 \\
\hline & \multirow{2}{*}{$50-69$} & Educación & & 1,69 & 1,10 & 2,58 \\
\hline & & Número de hijos & & 0,86 & 0,74 & 0,99 \\
\hline \multirow{2}{*}{ Examen clínico } & $30-49$ & \multicolumn{2}{|c|}{ Familiar diagnosticado con cáncer } & 3,50 & 1,66 & 7,35 \\
\hline & $50-69$ & \multicolumn{2}{|l|}{ Educación } & 1,68 & 1,24 & 2,27 \\
\hline \multirow{2}{*}{ Mamografía } & $30-49$ & \multicolumn{2}{|c|}{ Familiar diagnosticado con cáncer } & 2,81 & 1,22 & 6,47 \\
\hline & $50-69$ & \multicolumn{2}{|l|}{ Educación } & 1,50 & 1,12 & 2,00 \\
\hline
\end{tabular}

* Las variables introducidas en los modelos fueron: grado de escolaridad, estado civil, afiliación al sistema de salud, número de hijos, haber tenido un familiar con cáncer de seno y el puntaje sumatorio de conocimientos acerca del cáncer, factores de riesgo y síntomas.

encuentran la falta de síntomas, el olvido o descuido, y no haber recibido una recomendación médica. Por otro lado, los análisis bivariados y multivariados evidencian que tener un mayor nivel educativo se asoció con la realización de los exámenes de detección temprana.

La mayoría $(91,3 \%)$ de participantes considera que el cáncer de seno es curable si se detecta tempranamente, apreciación que es correcta, y al medir los conocimientos generales acerca de la enfermedad, se observa que, en la mayoría de las preguntas, más del $80 \%$ de las participantes tenían conocimientos adecuados. Resultados similares fueron reportados por Sánchez et $a l$. en Maracaibo, Venezuela, en donde más del $80 \%$ de las participantes respondieron acertadamente preguntas de conocimientos generales de cáncer de seno [10]. Sin embargo, nuestro estudio evidenció bajo conocimiento con relación a los factores de riesgo hormonal, como el presentar la menarquía antes de los 12 años (el 79,7 \% respondió incorrectamente) y la menopausia después de los 52 años (el 80,9\% respondió incorrectamente). En el caso de la menarquia, concuerda con los resultados de Sánchez et al., en el que más del $50 \%$ de las encuestadas manifestaban no conocer que la menarquia precoz representa un factor de riesgo para cáncer de seno, y difiere en el caso de la menopausia tardía, debido a que más del $50 \%$ de las venezolanas encuestadas sí reconocen la asociación del cáncer de seno con factores de riesgo como la menopausia tardía [10].

Aunque las mujeres tienen algunos conocimientos generales sobre el cáncer de seno y afirman que la enfermedad se puede detectar tempranamente, tan solo el $41,5 \%$ se ha practicado el examen clínico del seno y solo la mitad de ellas se lo ha hecho por chequeo.
Estos datos son ligeramente inferiores a los entregados por la ENDS del año 2015, que reporta que al $48 \%$ de las mujeres les han practicado el examen clínico de seno y el $65 \%$ de estas mujeres manifestaron que se lo hicieron por chequeo [3]. En el caso de la mamografía, las cifras son aún más alarmantes: en nuestro estudio, solo el 39,2 \% de las mujeres entre 50 y 69 años se ha efectuado la mamografía, mientras que a nivel nacional, según la ENDS, este porcentaje asciende a $63 \%$, y en Antioquia, a $53 \%$ [3]. El 70,1\% de las participantes expresó no habérsela hecho, debido a que no había una recomendación médica, datos explicables al tener en cuenta las bajas coberturas exigibles a las EPS tanto del régimen contributivo como del subsidiado, que, según la Resolución 3384 del 2000 y el Acuerdo 029 de 2011 [11,12], es de tan solo un $20 \%$, convirtiéndose esto en una importante barrera administrativa que afecta el acceso a la mamografía.

En comparación con la mamografía y el examen clínico, el autoexamen de seno es practicado por una mayor proporción de mujeres. En Colombia, según la ENDS de 2015, el $94 \%$ de las mujeres de 21 a 69 años de edad conoce el autoexamen de seno y el $72 \%$ se lo ha hecho, y entre quienes se lo han realizado, el $38 \%$ se lo hace cada mes [3]. Y aunque en nuestro estudio el conocimiento del autoexamen es $73,1 \%$ y únicamente el $21,8 \%$ se lo hace cada mes, el porcentaje de su práctica es similar, con un $69,9 \%$. Estas cifras superiores en la realización del autoexamen pueden deberse a que ha habido una mayor difusión por medios masivos de comunicación acerca de la importancia del autoexamen de seno. Sin embargo, esto contrasta con el conocimiento acerca de la técnica y las recomendaciones 
para la realización del autoexamen, pues solo el 28,1 \% de las encuestadas refiere que el autoexamen se debe efectuar después de la menstruación, y ninguna de las participantes describió la totalidad de los pasos recomendados para llevar a cabo el autoexamen [15].

Esto difiere con lo reportado por Vivas et al. en mujeres de Cúcuta, Colombia, donde encontraron que el $74 \%$ sabía que el autoexamen se debe hacer después de la menstruación, y el $52 \%$ conocía la técnica del autoexamen [13].

Al igual que en nuestro estudio, encuestas nacionales de Colombia, México y España coinciden en que un mayor nivel de educación está asociado con tasas más altas de mamografía, autoexamen y examen clínico $[3,14,15]$. Aunque la incidencia de la enfermedad es más alta en mujeres de un mayor nivel socioeconómico o mayor nivel educativo, la mortalidad es más alta en mujeres con menor educación o menores niveles socioeconómicos, ya que estas son diagnosticadas con la enfermedad en estadios más avanzados [16]. Así, los programas de detección temprana tienen la responsabilidad de diseñar estrategias para que las mujeres con baja educación puedan acceder a los exámenes y entender fácilmente cómo llevarlos a cabo. Se debe tener en cuenta que muchas de estas mujeres probablemente no sepan leer ni escribir; por ejemplo, en la muestra en Amalfi, el 35,5\% de las mujeres no tenía ninguna educación o no había terminado la primaria.

Dada la naturaleza de autorreporte de nuestra encuesta, las tasas de la práctica de la mamografía y el examen clínico del seno podrían presentar sesgos de memoria o de reporte, por lo que se sugiere que próximas investigaciones involucren a las EPS, con el fin de contrastar la información. Esta investigación es la primera de su naturaleza en el municipio de Amalfi y puede ser de mucha utilidad para los programas de promoción y prevención que se realicen allí o en localidades similares.

En conclusión, existe una disposición favorable hacia la detección temprana del cáncer de seno que puede servir para fortalecer los conocimientos acerca de este y de los exámenes, y motivar la práctica de la prevención y diagnóstico. Las mujeres necesitan educación acerca de los factores de riesgo, la adecuada técnica para la realización del autoexamen, y la importancia de hacerse la mamografía a partir de los 50 años, a pesar de no presentar ningún síntoma. De igual forma, las instituciones de salud y el personal de salud deben promover la práctica de la mamografía y las EPS deben garantizar su cobertura y el pago de los gastos asociados para su realización, pues el gasto por cuenta de las mujeres para su realización es elevado. En escenarios de recursos limitados, donde es necesario priorizar las poblaciones objeto de estrategias de promoción y prevención, se recomienda realizarlas en mujeres con bajos niveles educativos o pertenecientes al régimen subsidiado.

\section{Agradecimiento}

A la Alcaldía Municipal de Amalfi y sus dependencias de Secretaría de Salud y Secretaría de Planeación, y a la comunidad, por su colaboración y aceptación en la realización de las encuestas.

\section{Financiación}

La investigación fue financiada por el fondo del Centro de Investigación de la Facultad Nacional de Salud Pública, con código INV556D-17.

\section{Conflicto de interés}

Los autores declaramos que conocemos las posibles situaciones causales de conflicto de interés y damos fe de que no existe alguna relación con entidades o personas que posibiliten el sesgo en los resultados.

\section{Declaración de responsabilidad}

Todo lo expresado en el texto es responsabilidad de los autores y se exime a la Universidad de cualquier aspecto que infrinja la propiedad intelectual.

\section{Referencias}

1. Ferlay J, Ervik M, Lam F, Colombet M, Mery L, Piñeros M, Znaor A, Soerjomataram I, Bray F. Global Cancer Observatory: Cancer Today. Lyon, France: International Agency for Research on Cancer [internet]; 2018 [citado 21 feb. 2019]. Disponible en: https://gco.iarc.fr/today/online-analysistable $? \mathrm{v}=2018 \&$ mode $=$ cancer\&mode_population $=$ continents\&po pulation $=900 \&$ populations $=170 \&$ key $=$ asr $\&$ se $=0 \&$ cancer $=39 \&$ type $=1 \&$ statistic $=5 \&$ prevalence $=0 \&$ population_group $=0 \&$ ages group $\% 5 \mathrm{~B} \% 5 \mathrm{D}=0 \&$ ages group $\% 5 \mathrm{~B} \% 5 \mathrm{D}=17 \& \mathrm{nb}$ items $=5 \&$ group_cancer $=1 \&$ include_nmsc $=1 \&$ include_nmsc other $=1 \#$ collapse-group- $0-1$

2. Pardo C, de Vries E, Buitrago L, Gamboa O. Atlas de mortalidad por cáncer en Colombia. 4.a ed. Bogotá: Instituto Nacional de Cancerología; 2017.

3. Colombia, Ministerio de la Protección Social, Profamilia. Encuesta nacional de demografía y salud 2015. Bogotá [internet]; 2017 [citado 2017 abr. 10]. Disponible en: https://profamilia.org. co/investigaciones/ends/.

4. Kwok C, Lee MJ, Lee CF. Breast cancer perceptions and screening behaviours among Korean women in Australia. J. Immigr. Minor. Health. 2019;1-8. DOI: http://dx.doi.org/10.1007/s10903-01900876-8.

5. Kalliguddi S, Sharma S, Gore CA. Knowledge, attitude, and practice of breast self-examination amongst female IT professionals in Silicon Valley of India. J. Family. Med. Prim. 
Care. 2019;8(2):568-72. DOI: http://dx.doi.org/10.4103/jfmpc. jfmpc_315_18.

6. Wu TY, Lee J. Promoting breast cancer awareness and screening practices for early detection in low-resource settings. Eur. J. Breast. Health. 2019;15(1):18-25. DOI: http://dx.doi.org/10.5152/ ejbh.2018.4305.

7. Piñeros $\mathrm{M}$, Díaz $\mathrm{S}$, Poveda $\mathrm{C}$, et al. Manual para la detección temprana de cáncer de mama. Bogotá [internet]; 2015 [citado 2019 feb. 20]. Disponible: https://www.minsalud.gov.co/ Documentos\%20y\%20Publicaciones/Manual\%20para\%201a\%20 detecci $\% \mathrm{C} 3 \% \mathrm{~B} 3 \mathrm{n} \% 20$ temprana $\% 20 \mathrm{del} \% 20 \mathrm{c} \% \mathrm{C} 3 \% \mathrm{~A} 1 \mathrm{ncer} \% 20$ de $\% 20$ seno.pdf.

8. Castillo I, Bohórquez C, Palomino J, Elles L, Montero, L. Conocimientos, actitudes y prácticas sobre el autoexamen de mama en mujeres del área rural de Cartagena. Rev. U.D.C.A Act. \& Div. Cient. [Internet]. 2016; 19(1):5-14 [citado 2017 abr. 17]. Disponible en: http://www.scielo.org.co/pdf/rudca/v19n1/ v19n1a02.pdf.

9. Colombia. Ministerio de Salud. Resolución 8430, por la cual se establecen las normas científicas, técnicas y administrativas para la investigación en salud (1993 oct. 4).

10. Sánchez Urdaneta Y, Urdaneta Machado JR, Villalobos Inciarte N, et al. Conocimiento sobre cáncer de mamas y práctica del autoexamen de mamas en mujeres de edad mediana. Rev Venez Oncol [internet]. 2016; [citado 2017 abr. 11 ]; 28(1):37-51. Disponible en: http:// www.redalyc.org/pdf/3756/375643222006.pdf.
11. Colombia, Ministerio de Salud. Resolución 3384 por la cual se modifican parcialmente las resoluciones 412 y 1745 de 2000 y se deroga la Resolución 1078 de 2000 (2000 dic. 29).

12. Colombia,Comisión de Regulación en Salud (CRES). Acuerdo 029 de 2011 por el cual se sustituye el Acuerdo 028 de 2011 que define, aclara y actualiza integralmente el Plan Obligatorio de Salud (2011 dic. 30).

13. Vivas V, Torres N, Esguerra L, et al. Conocimientos, actitudes y prácticas de prevención de cáncer de mama. Ciencia y Cuidado [internet]. 2012 [citado 2017 abr. 14]; 9(2):43-51. Disponible en: https://revistas.ufps.edu.co/index.php/cienciaycuidado/article/ view/338.

14. Martín-López R, Hernández-Barrera V, De Andres AL, et al. Breast and cervical cancer screening in Spain and predictors of adherence. Eur. J. Cancer. Prev. 2010;19(3):239-45. DOI: http:// dx.doi.org/10.1097/CEJ.0b013e3283372125.

15. López-Carrillo L, Suárez-López L, Torres-Sánchez L. Detección del cáncer de mama en México: síntesis de los resultados de la Encuesta Nacional de Salud Reproductiva. Salud Pública Mex. 2009;51 Supl. 2:s345-9.

16. Bray F, McCarron P, Parkin DM. The changing global patterns of female breast cancer incidence and mortality. Breast Cancer Res. 2004;6(6):229-39. DOI: http://dx.doi.org/10.1186/bcr932. 